\title{
Genetic Analysis of the Twenty-One-Day Pregnancy Rate in US Holsteins Using an Ordinal Censored Threshold Model with Unknown Voluntary Waiting Period
}

\author{
Y. M. Chang, ${ }^{*}$ O. González-Recio,† K. A. Weigel, ${ }^{* 1}$ and P. M. Fricke* \\ *Department of Dairy Science, University of Wisconsin-Madison, Madison 53706 \\ †Departamento de Producción Animal Universidad Politécnica de Madrid, Madrid 28040, Spain
}

\begin{abstract}
Genetic variation in the number of 21-d opportunity periods required to achieve pregnancy after the voluntary waiting period (VWP) had passed was examined using 44,901 lactation records of 29,422 lactating Holstein cows on 61 large commercial dairy farms in the United States. Cows were allowed a maximum of 8 opportunity periods, and the cumulative percentages of cows that became pregnant by the end of the first, second, third, fourth, and fifth opportunity periods were $19,29,37,43$, and $47 \%$, respectively. In addition, $38 \%$ of records were censored because of culling or failure to achieve pregnancy after 8 opportunity periods. Mean days open was $128 \mathrm{~d}$ for complete records, whereas mean days to last service was $148 \mathrm{~d}$ for censored records. An ordinal censored threshold model was developed, in which duration of the VWP was estimated simultaneously with prediction of sire breeding values. The posterior mean of intraherd-year heritability for the number of 21-d opportunity periods required to achieve pregnancy was 0.06 , with a posterior standard deviation of 0.01. Posterior means for duration of the VWP ranged from 28 to $74 \mathrm{~d}$ postpartum among the 116 herd-parity classes represented in the study, whereas farmer-reported survey values for duration of the VWP ranged from 30 to $78 \mathrm{~d}$ postpartum. Sires' predicted transmitting abilities were computed, assuming an unknown VWP (i.e., estimated from the data), a VWP fixed at 60 d postpartum, or a VWP fixed at farmer survey values. Correlations among sire predicted transmitting abilities from different models were $\geq 0.98$, although some reranking occurred among top sires. In summary, the proposed model for genetic evaluation of female fertility can accommodate heterogeneity in duration of the VWP between herds, as well as heterogeneity that may arise within herds owing to management practices such as
\end{abstract}

Received June 8, 2006.

Accepted November 30, 2006.

${ }^{1}$ Corresponding author: kweigel@wisc.edu intentional delay of first insemination in high-producing cows or cows with poor body condition, and it can also accommodate censored records for nonpregnant cows.

Key words: days open, fertility, pregnancy rate, voluntary waiting period

\section{INTRODUCTION}

Improvement of female fertility in lactating dairy cows has become a key objective of national and international genetic selection programs in recent years. However, a lack of consensus exists among countries as to which traits should be considered, as noted by Jorjani (2005), who reported low genetic correlations among 28 measures of female fertility used in 14 different countries. Days to first service (DFS) measures the cow's ability to begin cycling and show visible heat after calving, whereas binary or categorical traits such as conception rate $(\mathbf{C R})$, nonreturn rate (NRR), or services per conception (SPC) measure the cow's ability to conceive and maintain the pregnancy. Composite traits such as calving interval (CI) or days open (DO) reflect both aspects of reproductive performance in a single measurement (Ferguson and Galligan, 1999).

Most of the aforementioned measures of female fertility suffer from one or more limitations. Measurements of DFS and DO can be influenced by hormonal synchronization or timed AI programs (Caraviello et al., 2006), as well as by intentional extension of the voluntary waiting period (VWP) of high-producing cows to achieve enhanced CR or increased milk yield (Tenhagen et al., 2003; Stevenson and Phatak, 2005). Measurements of $\mathrm{CR}$ are lacking in countries where pregnancy examination results are not reported routinely, and CR may be overestimated in cases in which ultrasound technology has been used for early pregnancy detection because of false positive diagnoses or yet to be observed embryonic losses. Although commonly used in many countries, NRR can be inflated if the assumption that the last reported insemination resulted in a pregnancy is violated. Censoring of records from nonpregnant animals 
is also a limitation for SPC, DO, and CI, the latter of which also suffers from a considerable time lag before data become available for genetic evaluation.

Nonetheless, CI, DO, or transformations thereof are routinely evaluated in many countries in which specific insemination data are not recorded, whereas DFS and NRR or SPC are routinely evaluated in many countries in which inseminations are reported without corresponding pregnancy examination results. In nearly every case, censoring of records from nonpregnant cows is ignored, either by excluding censored records or by fixing these observations at some arbitrary value. González-Recio et al. (2006) proposed 2 alternative models for the number of inseminations to conception that accommodated censoring (a censored linear model and a failure time model) and led to higher heritability estimates compared with models that excluded censored records or treated such records as complete. The effects of hormonal synchronization are also generally ignored, as is potential heterogeneity in duration of the VWP. Farm-specific management decisions may dictate differences in VWP because of parity, milk yield, BCS, or other factors (Caraviello et al., 2006). An intentional delay in time to first AI can be economically advantageous in high-yielding cows, as noted by Arbel et al. (2001), who reported increases in net returns of $\$ 0.19$ and $\$ 0.12 / \mathrm{d}$ in primiparous and multiparous cows with an extended calving interval, respectively. Furthermore, delays in time to first AI may improve CR when using hormonal synchronization protocols to submit cows for first service using timed AI, as reported by Tenhagen et al. (2003) and Stevenson and Phatak (2005). Thus, variation in duration of the VWP within farms attributable to management decisions should be considered in genetic evaluations, although information regarding such decisions may not be readily available.

The 21-d pregnancy rate (PR21) is defined as the percentage of eligible nonpregnant cows that become pregnant during a given 21-d interval, where eligibility is conferred by passing the producer-defined VWP. This measure of reproductive performance has become extremely popular among managers of large commercial dairy farms in the United States, especially those who use the Dairy Comp 305 herd management software program (Valley Agricultural Software, Tulare, CA).

The USDA-ARS Animal Improvement Programs Laboratory introduced national genetic evaluations for daughter pregnancy rate (DPR) in 2003 (VanRaden et al., 2004). The input to this genetic evaluation system is actually DO data, which are subsequently transformed to an approximation of PR21 using the formula: PR21 $=21 /(D O-V W P+11)$. Censored DO records of cows that are culled prior to a positive pregnancy diagnosis or are not yet pregnant at the time of evaluation are projected, using an upper limit of $250 \mathrm{~d}$ postpartum (VanRaden et al., 2004).

VanRaden (2003) noted that PR21 can be viewed as a sequence of binary variables (pregnant or nonpregnant) corresponding to each 21-d interval after the VWP has passed. However, some patterns of binary outcomes are not biologically plausible [e.g., $(0,1,0),(0,1,1)$, etc.], so it may be more logical to view PR21 as the number of opportunity periods required to achieve pregnancy after the VWP has passed. Although one might anticipate a loss of information when transforming DO, a continuous variable, to PR21, a categorical variable, it should be noted that DO is not biologically expressed on a continuous scale. For example, a cow that fails to conceive to an insemination at $80 \mathrm{~d}$ postpartum cannot achieve a DO phenotype of 85 or $90 \mathrm{~d}$, because her next opportunity to become pregnant will not occur until roughly $101 \mathrm{~d}$ postpartum. Likewise, if a farmer who uses a VWP of $50 \mathrm{~d}$ chooses not to inseminate a particular cow at $49 \mathrm{~d}$ postpartum, her next opportunity to achieve pregnancy will occur at approximately $70 \mathrm{~d}$ postpartum. Furthermore, genetic analysis of PR21 allows equivalent treatment of cows that are inseminated to standing heat and those that are subject to hormonal synchronization and timed AI programs, because it does not matter whether conception events are concentrated on a single day or distributed randomly throughout the 21-d interval.

Current genetic evaluations for US dairy cattle assume a constant VWP for all cows. Heterogeneity in duration of the VWP between herds was reported by Miller et al. (2005), who used the 10th percentile of DFS as a proxy for VWP. More important, a recent survey of managers of large commercial dairy farms indicated heterogeneity of the VWP within herds, because many routinely extend the VWP for specific groups of cows according to milk yield, BCS, and other factors (Caraviello et al., 2006).

The primary objective of the present study was to develop a methodology for an ordinal censored threshold analysis of PR21, defined as the number of opportunity periods needed to achieve pregnancy, with unknown VWP parameters estimated from the data. The secondary objective was to apply the aforementioned ordinal censored threshold model to an example field data set with VWP fixed at $60 \mathrm{~d}$ for all cows, fixed at herd-parity-specific values reported in a farmer survey, or estimated from the data.

\section{MATERIALS AND METHODS}

\section{Data}

The present study used data from cows that calved from January 2000 to August 2004 on large commercial 
dairy farms in the Alta Genetics (Watertown, WI) Advantage Progeny Testing Program, which provided parity-specific VWP information in a reproductive management survey (Caraviello et al., 2006). These herds, which were located throughout the United States, had a mean herd size of $613 \pm 46$ cows, a mean quantity of milk sold per cow per day of $34.5 \pm 0.3 \mathrm{~kg}$, a mean culling rate of $34 \pm 1 \%$, and a mean calving interval of $13.8 \pm 0.1$ mo (Caraviello et al., 2006). It is important to note than many herds did not enroll in the Advantage Program until 2002 or 2003, and that the number of cows available for the present study was further reduced by excluding cows with unknown or non-AI sires (the percentage of unknown sires was high, because many herds had recently expanded with purchased animals). Insemination records and pregnancy examination results were available for all herds, and these were obtained from the on-farm Dairy Comp 305, PCDART (DRMS, Raleigh, NC), or DHI-Plus (DHI-Provo, UT) management software programs.

For complete records $(\mathrm{n}=27,711)$, which ended in a positive pregnancy diagnosis (within $75 \mathrm{~d}$ of the insemination), DO was computed and was restricted to a range of 35 to 400 d postpartum. For censored records ( $\mathrm{n}=$ 17,190 ), which ended in a negative pregnancy diagnosis or an insemination without a corresponding pregnancy examination (within $75 \mathrm{~d}$ ), DO could not be computed and was therefore replaced by days to last service (DLS), which was also restricted to a range of 35 to $400 \mathrm{~d}$ postpartum. Reasons for censoring included culling prior to a positive pregnancy diagnosis, failure to achieve pregnancy by the end of the eighth opportunity period, movement to a "clean-up" pen for natural service mating, designation as "do not breed," and lack of a pregnancy examination following the last insemination. For all animals, DFS was restricted to a range of 25 to $175 \mathrm{~d}$ postpartum. The final data set consisted of 44,901 lactation records from 29,422 Holstein cows in 61 herds. The example analysis described herein considered specific VWP parameters for each herd-parity class (primiparous or multiparous), and 3 different values were considered: 1) a VWP fixed at $60 \mathrm{~d}$ postpartum for all cows, regardless of herd or parity; 2) a VWP fixed at farmer-reported survey values for each herd-parity class, or 3) a VWP estimated from DFS data for each herd-parity class in a Bayesian analysis (described below).

\section{Statistical Model}

Censored Threshold Model. Assume that the number of opportunity periods required to achieve pregnancy after the VWP has passed is calculated as

$$
y_{i(k)}=\operatorname{ceil}\left[\left(D O_{i(k)}-V W P_{k}\right) / 21\right],
$$

where $y_{i(k)}$ is the number of opportunity intervals for cow $i$ within herd-parity $k\left(y_{i(k)}=1,2, \ldots, J\right.$, where $J \leq$ 8); $D O_{i(k)}$ is the number of DO for cow $i$ within herdparity $k ; V W P_{k}$ is the VWP for herd-parity $k$, and ceil(.) is a function that returns the smallest positive integer that is greater than or equal to the input argument. Based on equation [1], the probability model for PR21, the number of opportunity periods required to achieve pregnancy after the VWP has passed, can be written as

$$
\begin{gathered}
\operatorname{prob}\left(y_{i(k)} \mid \beta, \mathbf{h}, \mathbf{s}, \mathbf{T}, D O_{i(k)}, V W P_{k}\right)= \\
\Phi\left[T_{y_{i(k)}}-\left(\mathbf{x}_{i(k)}^{\prime} \beta+\mathbf{z}_{h, i(k)}^{\prime} \mathbf{h}+\mathbf{z}_{s, i(k)}^{\prime} \mathbf{s}\right)\right] \\
-\Phi\left[T_{y_{i(k)}-1}-\left(\mathbf{x}_{i(k)}^{\prime} \beta+\mathbf{z}_{h, i(k)}^{\prime} \mathbf{h}+\mathbf{z}_{s, i(k)}^{\prime} \mathbf{s}\right)\right]
\end{gathered}
$$

where $y_{i(k)}$ is the number of opportunity periods, $\Phi(\cdot)$ is the standard cumulative normal distribution function, and $\mathbf{T}=\left[\mathrm{T}_{0}, \mathrm{~T}_{1}, \mathrm{~T}_{2}, \cdots, \mathrm{T}_{8}\right]^{\prime}$ is the vector of unknown thresholds. The thresholds must satisfy the condition $-\infty=\mathrm{T}_{0} \leq \mathrm{T}_{1} \leq \mathrm{T}_{2} \leq \cdots \leq \mathrm{T}_{8}=\infty$. The first threshold $\mathrm{T}_{1}$ is set to zero, because this parameter cannot be identified in a probit analysis. The vector $\boldsymbol{\beta}$ included effects of parity (4 levels), year-season (18 levels), and month of calving (12 levels). Furthermore, $\mathbf{h}$ contained herdyear effects (211 levels), $\mathbf{s}$ was the vector of sire transmitting abilities (5719 levels), and $\mathbf{x}, \mathbf{z}_{\mathrm{h}}$, and $\mathbf{z}_{\mathrm{s}}$ were incidence matrices.

This concept accommodates situations in which records were censored at the last observed point. If an observation was censored at the $j$ th opportunity interval, with unknown pregnancy status, then its corresponding liability must be larger than $\mathrm{T}_{j-1}$. The probability that the observation was censored at the $j$ th category is

$$
\begin{gathered}
\operatorname{prob}\left(y_{i(k)}=j \text { censored } \mid \beta, \mathbf{h}, \mathbf{s}, \mathbf{T}, D O_{i(k)}, V W P_{k}\right)= \\
1-\Phi\left[T_{\left.y_{i(k)}\right)^{-1}}-\left(\mathbf{x}_{i(k)}^{\prime} \beta+\mathbf{z}_{h, i(k)}^{\prime} \mathbf{h}+\mathbf{z}_{s, i(k)}^{\prime} \mathbf{s}\right)\right] .
\end{gathered}
$$

The joint probability of $N$ complete and censored PR21 observations, given DO records, VWP information, location effects, and thresholds, is

$$
\begin{gathered}
\operatorname{prob}(\mathbf{y} \mid \beta, \mathbf{h}, \mathbf{s}, \mathbf{T}, \mathbf{D O}, \mathbf{V W P}, \boldsymbol{\delta})= \\
\prod_{i=1}^{N}\left\{\Phi\left[T_{y_{i(k)}}-\left(\mathbf{x}_{i(k)}^{\prime} \beta+\mathbf{z}_{h, i(k)}^{\prime} \mathbf{h}+\mathbf{z}_{s, i(k)}^{\prime} \mathbf{s}\right)\right]\right. \\
\left.-\Phi\left[T_{y_{i(k)}-1}-\left(\mathbf{x}_{i(k)}^{\prime} \beta+\mathbf{z}_{h, i(k)}^{\prime} \mathbf{h}+\mathbf{z}_{s, i(k)}^{\prime} \mathbf{s}\right)\right]\right\}^{1-\delta_{i}} \\
\left\{1-\Phi\left[T_{y_{i(k)}-1}-\left(\mathbf{x}_{i(k)}^{\prime} \beta+\mathbf{z}_{h, i(k)}^{\prime} \mathbf{h}+\mathbf{z}_{s, i(k)}^{\prime} \mathbf{s}\right)\right]\right\}^{\delta_{i}},
\end{gathered}
$$


where $\delta$ is the vector of censoring indicators, such that $\delta_{i}=0$ for a record that is complete (status known) and 1 for a record that is censored (status unknown).

Bounded uniform priors were assigned to each of the elements of $\beta$ as $\beta \sim U\left(\beta_{\min }, \beta_{\max }\right)$, with $\beta_{\min }=-9,999$ and $\beta_{\max }=9,999$. A multivariate normal prior was used for herd-year effects as $\mathbf{h} \sim N\left(0, \mathbf{I} \sigma_{h}^{2}\right)$, where $\mathbf{I}$ is an identity matrix of order $211 \times 211$, and $\sigma_{h}^{2}$ is the between-herd-year variance. The vector of sire effects was assigned a multivariate normal prior distribution as $\mathbf{s}$ $\sim N\left(0, \mathbf{A} \sigma_{s}^{2}\right)$, where $\sigma_{s}^{2}$ is the variance between sire transmitting abilities, and $\mathbf{A}$ is the additive relationship matrix between male ancestors. Independent scaled inverse $\chi^{2}$ prior distributions were used for $\sigma_{h}^{2}$ and $\sigma_{s}^{2}$ as

$$
\begin{gathered}
\sigma_{h}^{2} \sim v_{h} s_{h}^{2} \chi^{-2}\left(v_{h}, s_{h}^{2}\right) \text { and } \\
\sigma_{s}^{2} \sim v_{s} s_{s}^{2} \chi^{-2}\left(v_{s}, s_{s}^{2}\right),
\end{gathered}
$$

respectively, where $\nu_{h}=\nu_{s}=3$ are the degrees of belief parameters, and $\mathrm{s}_{h}^{2}=0.1$ and $\mathrm{s}_{s}^{2}=0.05$ are the scale parameters, respectively. These hyperparameters are used to reflect weak and proper priors (other scale parameters were examined, but there was no change in the posterior distributions). Because the first threshold was fixed at 0 , the remaining 6 thresholds associated with the 8 opportunity periods required to achieve pregnancy after the VWP had passed were assumed to be distributed as order statistics from a uniform distribution with the restriction $0 \leq \mathrm{T}_{2} \leq \cdots \leq \mathrm{T}_{7}$.

Although the VWP is often assumed to be predetermined by the herd management protocol, individual herdsmen or breeding technicians sometimes deviate from the protocol. For example, a cow observed in standing estrus at $50 \mathrm{~d}$ postpartum may be inseminated even if the stated VWP was set at $60 \mathrm{~d}$ postpartum. Therefore, Miller et al. (2005) suggested that the 10th percentile of DFS could serve as an approximation of VWP. In this study, a bounded uniform prior was used, using the minimum DFS observation in herd-parity $k$ as the lower bound and the 25th percentile of DFS in herdparity $k$ as the upper bound for $V W P_{k}$. Draws from posterior distributions, except for the thresholds and VWP parameters, were obtained using a Gibbs sampler, after augmentation of the joint posterior density with unobserved liabilities corresponding to the number of opportunity intervals (Sorensen and Gianola, 2002).

The method of Albert and Chib (1997) was used for sampling thresholds, where a natural log transformation of the thresholds was adopted as

$$
\begin{gathered}
\alpha_{1}=0 \text { and } \\
\alpha_{j}=\ln \left(\mathrm{T}_{j}-\mathrm{T}_{j-1}\right),
\end{gathered}
$$

where $j=2, \ldots, J-1$. Thresholds were obtained as

$$
\mathrm{T}_{j}=\sum_{i=1}^{j} \exp \left(\alpha_{i}\right), 1 \leq j \leq \mathrm{J}-1 .
$$

Note that $\alpha_{j}$ does not have any constraints on order, as in the case of the standard parameterization of thresholds. A Metropolis algorithm was used to obtain each of the $\alpha$ 's by sampling from a normal proposal distribution with mean equal to the value from the previous iteration and standard deviation equal to 0.002 to ensure a reasonable acceptance rate.

As noted earlier, the number of opportunity intervals was calculated as

$$
y_{i(k)}=\operatorname{ceil}\left(\frac{D O_{i(k)}-V W P_{k}}{21}\right)
$$

Therefore, the full conditional posterior of the VWP for all records in herd-parity $k$ can be written as

$$
\begin{gathered}
p\left(V W P_{k} \mid \beta, \mathbf{h}, \mathbf{s}, \mathbf{T}, \mathbf{D O}, \mathbf{D F S}, \boldsymbol{\delta}\right) \propto \prod_{i=1}^{N_{k}} \\
\left\{\Phi\left[T_{y_{i(k)}}-\left(x_{i(k)}^{\prime} \beta+z_{h, i(k)}^{\prime} \mathbf{h}+z_{s, i(k)}^{\prime} \mathbf{s}\right)\right]\right. \\
\left.-\Phi\left[T_{\left.y_{i(k)}\right)^{-1}}-\left(x_{i(k)}^{\prime} \beta+z_{h, i(k)}^{\prime} \mathbf{h}+z_{s, i(k)}^{\prime} \mathbf{S}\right)\right]\right\}^{1-\delta_{i}} \\
\left\{1-\Phi\left[T_{y_{i(k)}-1}-\left(x_{i(k)}^{\prime} \beta+z_{h, i(k)}^{\prime} \mathbf{h}+z_{s, i(k)}^{\prime} \mathbf{s}\right)\right]\right\}^{\delta_{i}} .
\end{gathered}
$$

Here, $N_{k}$ is the number of cows in herd-parity $k$, and $V W P_{k}$ entered the kernel density through its ceil transformation, $y_{i(k)}$. The posterior distribution of VWP does not have a closed form even after the introduction of liability. A single-site Metropolis algorithm can be used to sample the VWP from its posterior distribution conditional on the new candidate $V W P_{k}^{*}$, satisfying the prior boundary of $\left[V_{k(\min )}, V_{k(\max )}\right]$. where $V_{k(\min )}$ is the minimum of DFS, and $V_{k(\max )}$ is the 25 th percentile of DFS in herd-parity $k$, respectively. The probability of accepting $V W P_{k}^{*}$ as a new draw from its posterior can be calculated as

$$
\pi_{k}=\min \left(1, \frac{l\left(V W P_{k}^{*} \mid \beta, \mathbf{h}, \mathbf{s}, \mathbf{T}, \mathbf{D O}, \mathbf{D F S}, \boldsymbol{\delta}\right)}{l\left(V W P_{k}^{[t-1]} \mid \beta, \mathbf{h}, \mathbf{s}, \mathbf{T}, \mathbf{D O}, \mathbf{D F S}, \boldsymbol{\delta}\right)}\right),
$$

where $V W P_{k}^{[t-1]}$ is the value of $V W P_{k}$ at the $[t-1]$ th iteration of the sampler.

Convergence Diagnostics. The method of Raftery and Lewis (1992) and visual inspections of trace plots were used to assess the number of iterations and the required length of burn-in. A single long chain of 150,000 iterations was used, and the initial 50,000 sam- 


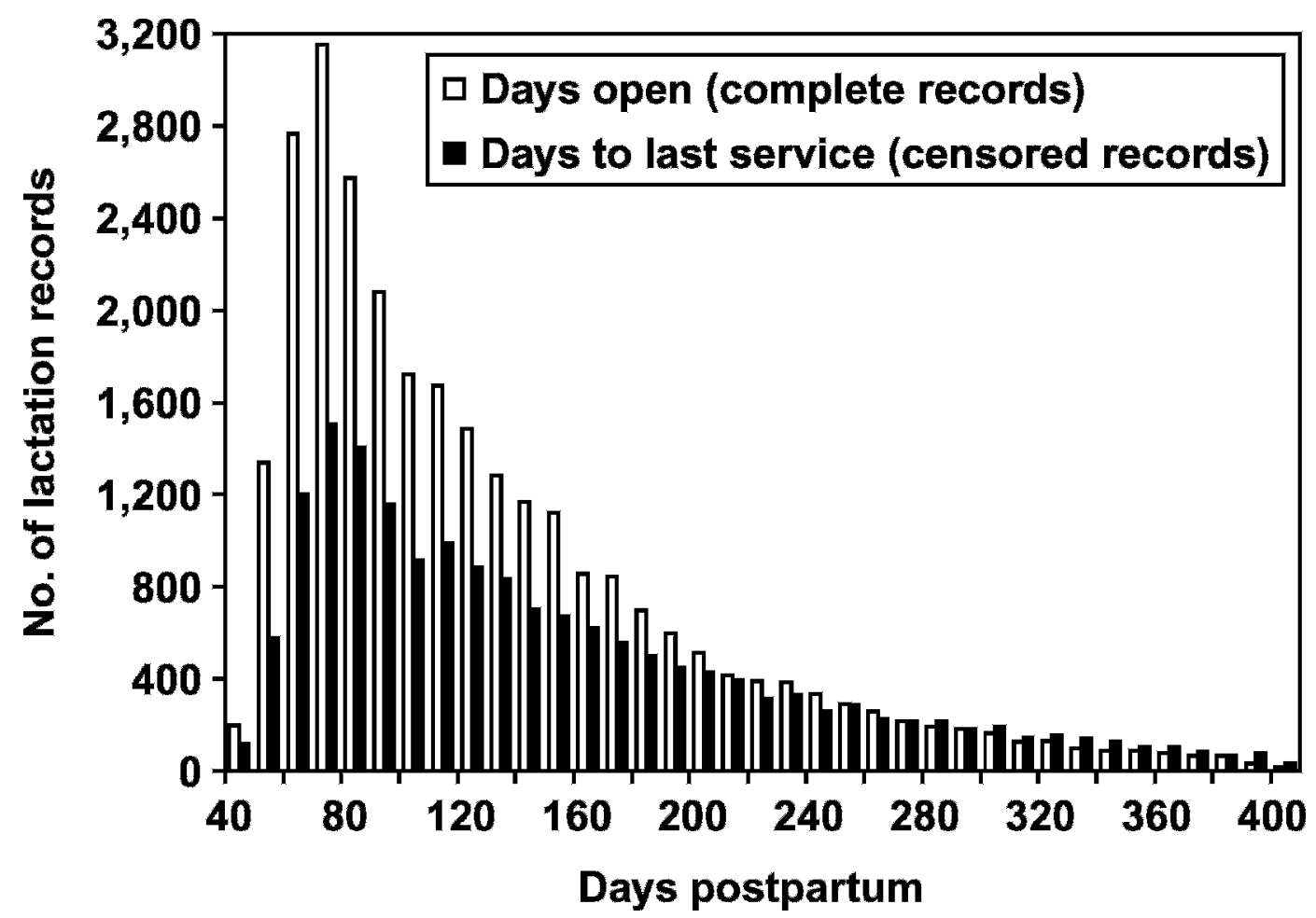

Figure 1. Distribution of days open for 27,711 complete lactation records (i.e., records that ended in a positive pregnancy diagnosis), and days to last service for 17,190 censored records (i.e., records that ended in a negative pregnancy diagnosis or an insemination without a corresponding pregnancy examination).

ples were discarded as burn-in. Inferences were based on the following 100,000 samples. The acceptance rate for the Metropolis algorithm was $40 \%$.

\section{RESULTS AND DISCUSSION}

The distributions of DO for complete records and DLS for censored records are shown in Figure 1. Mean DO for complete records and DLS for censored records were 128 and $148 \mathrm{~d}$, respectively, and distributions for both variables were skewed to the right. The distribution of herd-parity VWP parameters reported by herd managers in the farmer survey is shown in Figure 2. There were $24.2 \%$ of herd-parity classes with reported VWP less than $50 \mathrm{~d}$, an additional $37.1 \%$ with VWP between 50 and $59 \mathrm{~d}$, and $12.9 \%$ with VWP greater than $60 \mathrm{~d}$. Based on these responses, application of a fixed VWP for all animals does not seem reasonable. For $41.4 \%$ of herd-parity classes, the 10th percentile of DFS observations was less than the farmer-reported survey VWP, which seems to indicate a lack of compliance with the breeding protocols in some herds. Figure 2 also shows the distribution of estimated posterior means of herdparity VWP parameters. The mean absolute difference between posterior mean VWP and $60 \mathrm{~d}$ was approxi- mately $10 \mathrm{~d}$, whereas the majority of farmer-reported VWP values were less than the corresponding posterior means. This is illustrated in detail in Figure 3, which contains ordered differences between posterior mean VWP and $60 \mathrm{~d}$, and between posterior mean VWP and farmer survey VWP, for each of the 116 herd-parity classes represented in the study. The majority of estimated 95\% credible intervals for VWP (not shown) covered the corresponding farmer-reported VWP values. Differences between posterior mean VWP and $60 \mathrm{~d}$ postpartum were distributed more or less evenly about zero, although several herd-parity classes had large negative deviations. On the other hand, the mean of the posterior distribution of the VWP parameter, which had lower and upper bounds of the minimum and 25th percentile of DFS observations within a given herd-parity class, respectively, tended to be greater than farmer survey VWP for most herd-parity classes. Other bounds for the distribution of VWP could be considered in future studies, such as the minimum and 20th percentile of DFS, but the impact of such a change would probably be minimal.

Table 1 has the distribution of DO for complete records and DLS for censored records across opportunity periods for each of 3 VWP scenarios: fixed at $60 \mathrm{~d}$ post- 


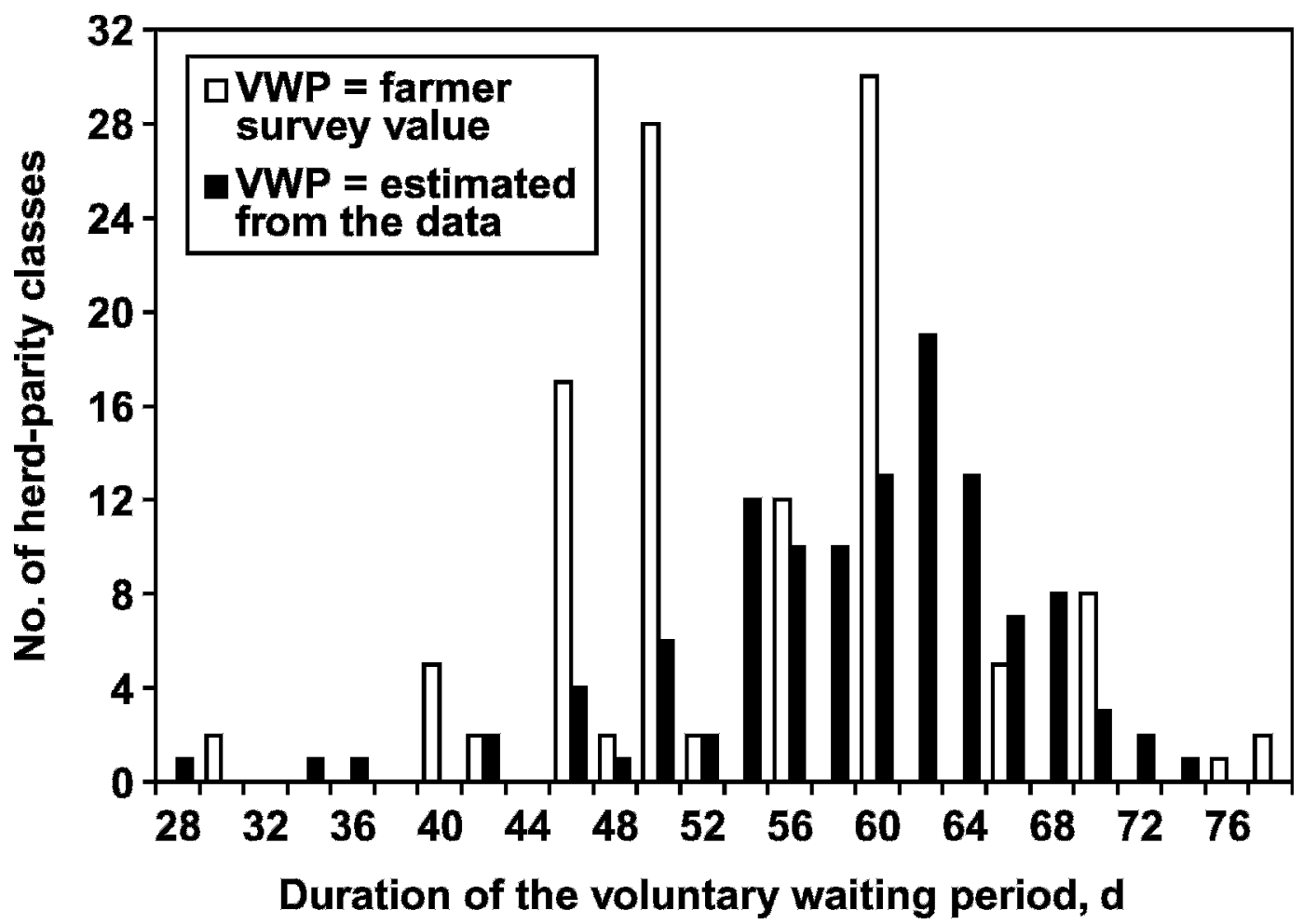

Figure 2. Distribution of voluntary waiting period (VWP) parameters in each of 116 herd-parity classes as estimated by the posterior mean in a censored ordinal threshold model or as reported in the farmer surveys.

partum, fixed at farmer survey values, or estimated from the data. Overall, $61.7 \%$ of records ended in pregnancy, and $38.3 \%$ were censored. When averaged across the 3 VWP scenarios, the cumulative proportion of cows that became pregnant was approximately $19 \%$ by the end of the first opportunity period, $29 \%$ by the end of the second period, $37 \%$ by the end of the third period, $43 \%$ by the end of the fourth period, $47 \%$ by the end of the fifth period, $51 \%$ by the end of the sixth period, and $54 \%$ by the end of the seventh period. An additional $8 \%$ became pregnant during or after the eighth period, whereas $38 \%$ of cows were nonpregnant or were culled from the herd before a positive pregnancy diagnosis.

Some cows were inseminated before the assumed, stated, or estimated VWP. When the across-herd-parity VWP was fixed at $60 \mathrm{~d}$ postpartum, $6.8 \%$ of cows became pregnant before the first interval, whereas $2.9 \%$ of records were censored at the beginning of the first interval. Corresponding percentages of pregnant and censored records that occurred before the first interval were 3.9 and $1.9 \%$, respectively, when within-herd-parity VWP parameters were fixed at farmer survey values and 5.6 and $2.4 \%$, respectively, when within-herd-parity VWP parameters were estimated from the data. In practice, it seems preferable to allow a small percentage of inseminations to precede the assumed, stated, or estimated VWP, rather than to base this parameter on a handful of outlier records that may represent recording errors or noncompliance with the herd's breeding protocol.

The posterior means of thresholds, that is, the points at which further increases in the cow's underlying propensity for poor fertility (on a continuous scale) translate into the need for an additional opportunity period (on a categorical scale), for the 3 VWP scenarios are given in Table 2. Posterior means were $0.35,0.63,0.85$, $1.05,1.22$, and 1.36 for the second, third, fourth, fifth, sixth, and seventh thresholds, respectively, when VWP parameters were estimated from the data. Corresponding posterior standard deviations for these thresholds ranged from 0.005 to 0.009 . All estimated thresholds using survey VWP were larger than thresholds estimated with VWP fixed at $60 \mathrm{~d}$ postpartum or VWP estimated from the data, because the starting point for the first opportunity period tended to be earlier in the survey VWP analysis.

Posterior means of the parity effects (not shown) were $0.13,0.19$, and 0.27 for parity 2 , parity 3 , and parity $\geq 4$, respectively, using first-parity records as a reference. Although there is no straightforward transformation from the liability scale to a probability scale, results indicated that older cows tended to require a greater 


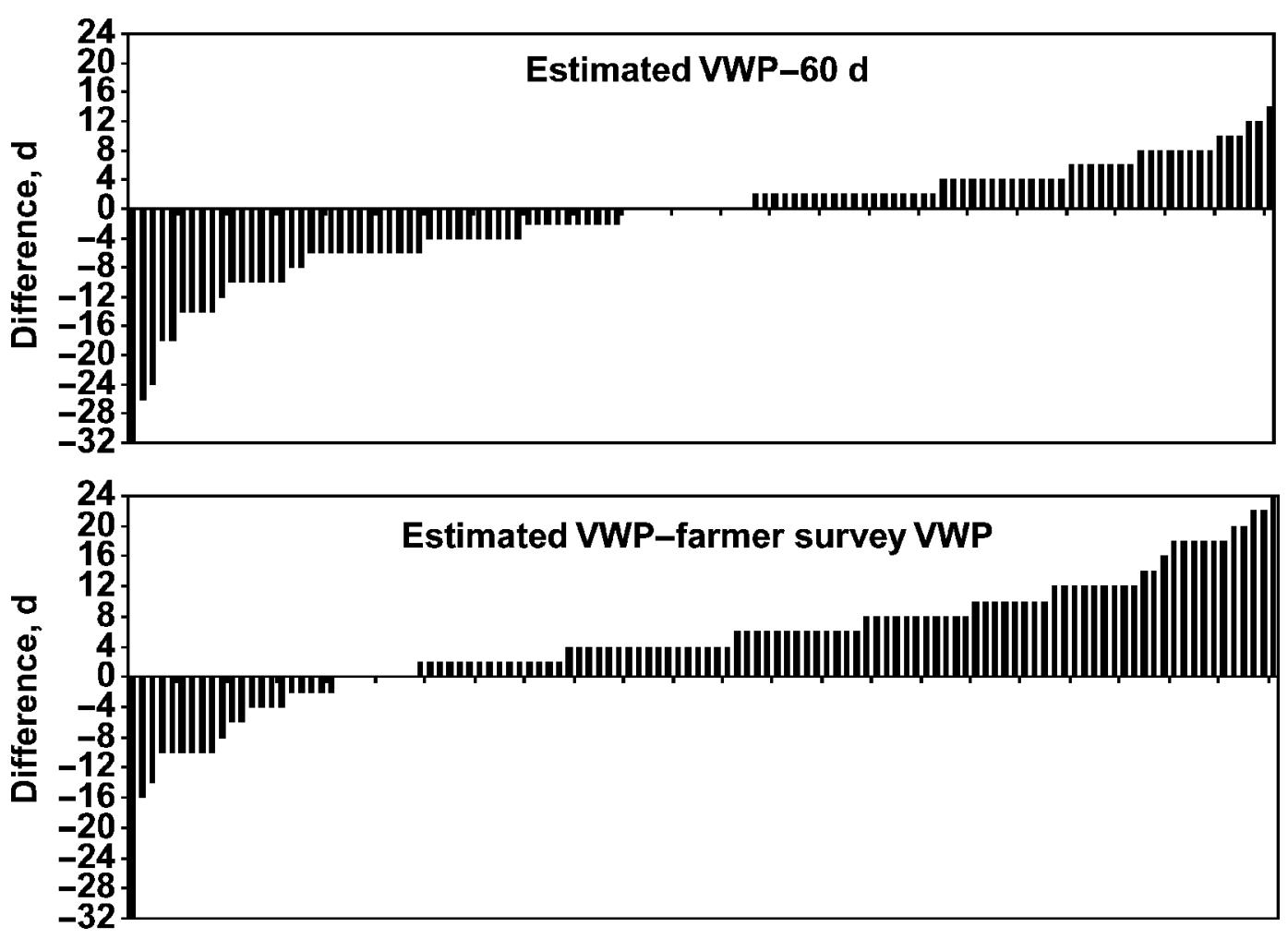

Figure 3. Ordered differences between the estimated posterior mean for the voluntary waiting period (VWP) and a fixed VWP of $60 \mathrm{~d}$ postpartum (top graph) or between the estimated posterior mean for the VWP and the farmer survey value (bottom graph) for each of 116 herd-parity classes.

Table 1. Percentages of pregnant and censored records in each 21-d opportunity period, based on a voluntary waiting period (VWP) fixed at $60 \mathrm{~d}$ postpartum for all cows, a herd-parity-specific VWP fixed at farmer survey values, or a herd-parity-specific VWP estimated from the data

\begin{tabular}{lcrrrrrrrrr}
\hline & \multicolumn{7}{c}{ Opportunity period } & \\
\cline { 2 - 8 } VWP & $\begin{array}{c}\text { Pregnancy } \\
\text { status }\end{array}$ & \multicolumn{1}{c}{$\leq 1$} & \multicolumn{1}{c}{2} & \multicolumn{1}{c}{3} & 4 & 5 & 6 & 7 & $\geq 8$ & Total \\
\hline Across-herd, fixed & Pregnant & 20.6 & 9.5 & 7.5 & 5.8 & 4.6 & 3.5 & 2.5 & 7.8 & 61.7 \\
at 60 d postpartum & Censored & 9.6 & 5.3 & 4.3 & 3.7 & 3.0 & 2.4 & 2.0 & 7.9 & 38.3 \\
Herd-parity-specific, fixed & Pregnant & 17.5 & 10.2 & 7.7 & 6.3 & 5.0 & 3.7 & 2.8 & 8.4 & 61.7 \\
at farmer survey values & Censored & 8.4 & 5.5 & 4.5 & 3.8 & 3.1 & 2.6 & 2.1 & 8.3 & 38.3 \\
Herd-parity-specific, & Pregnant & 20.1 & 9.1 & 7.6 & 5.9 & 4.7 & 3.6 & 2.6 & 8.0 & 61.7 \\
estimated from the data & Censored & 9.7 & 4.9 & 4.5 & 3.6 & 3.0 & 2.4 & 2.1 & 8.0 & 38.3 \\
\hline
\end{tabular}

Table 2. Posterior means of thresholds separating successive 21-d opportunity periods, based on a voluntary waiting period (VWP) fixed at $60 \mathrm{~d}$ postpartum for all cows, a herd-parity-specific VWP fixed at farmer survey values, or a herd-parity-specific VWP estimated from the data ${ }^{1}$

\begin{tabular}{lccccccc}
\hline & \multicolumn{5}{c}{ Threshold number } \\
\cline { 2 - 8 } VWP & 1 & 2 & 3 & 4 & 5 & 6 & 7 \\
\hline Across-herd, fixed at 60 d postpartum & 0.000 & 0.36 & 0.63 & 0.85 & 1.05 & 1.22 & 1.36 \\
Herd-parity-specific, fixed at farmer survey values & 0.000 & 0.41 & 0.69 & 0.93 & 1.14 & 1.31 & 1.46 \\
Herd-parity-specific, estimated from the data & 0.000 & 0.35 & 0.63 & 0.85 & 1.05 & 1.22 & 1.36 \\
\hline
\end{tabular}

${ }^{1}$ Note that the first threshold was constrained to zero in all analyses. 
Table 3. Posterior means (SD in parentheses) of herd-year variance $\left(\sigma_{h}^{2}\right)$, sire variance $\left(\sigma_{s}^{2}\right)$, heritability $\left[4 \sigma_{s}^{2} /\left(1+\sigma_{s}^{2}+\sigma_{h}^{2}\right)\right]$, and intraherd-year heritability $\left[4 \sigma_{s}^{2} /\left(1+\sigma_{s}^{2}\right)\right]$ for the number of 21-d opportunity periods needed to achieve pregnancy, based on a voluntary waiting period (VWP) fixed at $60 \mathrm{~d}$ postpartum for all cows, a herd-parity-specific VWP fixed at farmer survey values, or a herd-parity-specific VWP estimated from the data

\begin{tabular}{lccc}
\hline & \multicolumn{2}{c}{ VWP } \\
\cline { 2 - 4 } & $\begin{array}{c}\text { Across-herd, } \\
\text { fixed at } 60 \mathrm{~d} \\
\text { postpartum }\end{array}$ & $\begin{array}{c}\text { Herd-parity-specific, } \\
\text { fixed at farmer } \\
\text { survey values }\end{array}$ & $\begin{array}{c}\text { Herd-parity-specific, } \\
\text { estimated from } \\
\text { the data }\end{array}$ \\
\hline Parameter & $0.119(0.014)$ & $0.120(0.014)$ & $0.125(0.015)$ \\
Sire variance & $0.015(0.003)$ & $0.015(0.003)$ & $0.015(0.003)$ \\
Heritability & $0.053(0.009)$ & $0.054(0.009)$ & $0.053(0.009)$ \\
Intraherd-year heritability & $0.059(0.011)$ & $0.060(0.011)$ & $0.060(0.010)$ \\
\hline
\end{tabular}

number of opportunity periods to achieve pregnancy than younger cows. This could represent a direct consequence of aging or an indirect consequence of higher daily milk yield among older animals (Dematawewa and Berger, 1998; Abdallah and McDaniel, 2000; Dechow et al., 2004). Primiparous cows have been reported to have greater conception rates than multiparous cows in several recent studies (Stevenson et al., 2003; Chebel et al., 2004; Sterry et al., 2006).

Season of calving has been identified as an important factor with regard to DO, PR21, and other measures of reproductive performance. In agreement with previous studies (e.g., de Vries et al., 2005; de Vries and Risco, 2005), cows that calved in the spring and fall in the present study tended to require fewer opportunity periods to achieve pregnancy. Oseni et al. (2003) noted that distributions of DO observations tended to be bimodal for some calving months, presumably because of postponed breeding during the hot season or depressed fertility as a result of heat stress, and future studies should consider heterogeneity of the VWP according to season of calving.

Posterior means and corresponding standard deviations of sire variance, herd-year variance, and heritability parameters are shown in Table 3 . All posterior distributions were symmetric and unimodal. The posterior mean of the herd-year variance with VWP estimated from the data $(0.125)$ was slightly greater than when VWP was fixed at $60 \mathrm{~d}(0.119)$ or when VWP were fixed at farmer survey values (0.120). Posterior means of heritability and intraherd-year heritability parameters ranged from 0.053 to 0.054 and from 0.059 to 0.060 , respectively. In a previous study, Oseni et al. (2004) examined the effects of different editing criteria for DO, as well as the effects of different fixed VWP parameters, on genetic parameters for PR21 (approximated from DO) in US Holsteins. They found that heritability of PR21 increased as the upper limit for DO increased. Furthermore, they noted that the heritability of PR21 ranged more than 2 -fold, between 0.027 to 0.057 , in different states when the assumed VWP parameters ranged between 50 and $120 \mathrm{~d}$ postpartum.

Sire PTA for the 3 different VWP scenarios (fixed at $60 \mathrm{~d}$ postpartum, fixed at farmer survey values, or estimated from the data) tended to be similar, with rank correlations $\geq 0.98$. However, as shown in Figure 4 , the proportion of selected sires in common when selection was based on PTA with a fixed VWP of $60 \mathrm{~d}$ and when selection was based on PTA with VWP estimated from the data ranged from 0.93 to 0.94 when the top 5 to $35 \%$ of sires were chosen. Likewise, the proportion of selected sires in common when selection was based on PTA with VWP fixed at farmer survey values and when selection was based on PTA with VWP estimated from the data ranged from 0.87 to 0.96 . Overall, sire rankings with VWP fixed at $60 \mathrm{~d}$ postpartum more closely resembled rankings with VWP estimated from the data. However, this does not imply that a fixed VWP is preferable to farmer survey values, because the increased correlations observed herein may have occurred simply because the arbitrary population-wide VWP parameter chosen in this study (60 d) was very close to the average posterior mean VWP across herdparity classes.

Figure 5 demonstrates the relationship between sire PTA with VWP estimated from the data in the present study and sire PTA for DPR from the May 2006 genetic evaluation carried out by the USDA-ARS Animal Improvement Programs Laboratory. Among the limited number of sires $(n=71)$ with at least 100 daughters in both data sets, the product-moment correlation between sire PTA was -0.71, indicating that daughters of sires with positive PTA for DPR tended to require fewer opportunity periods to achieve pregnancy in the present study, whereas daughters of sires with negative PTA for DPR tended to require more opportunity periods.

\section{CONCLUSIONS}

The present study proposes a theoretically appealing and computationally feasible approach to genetic evalu- 


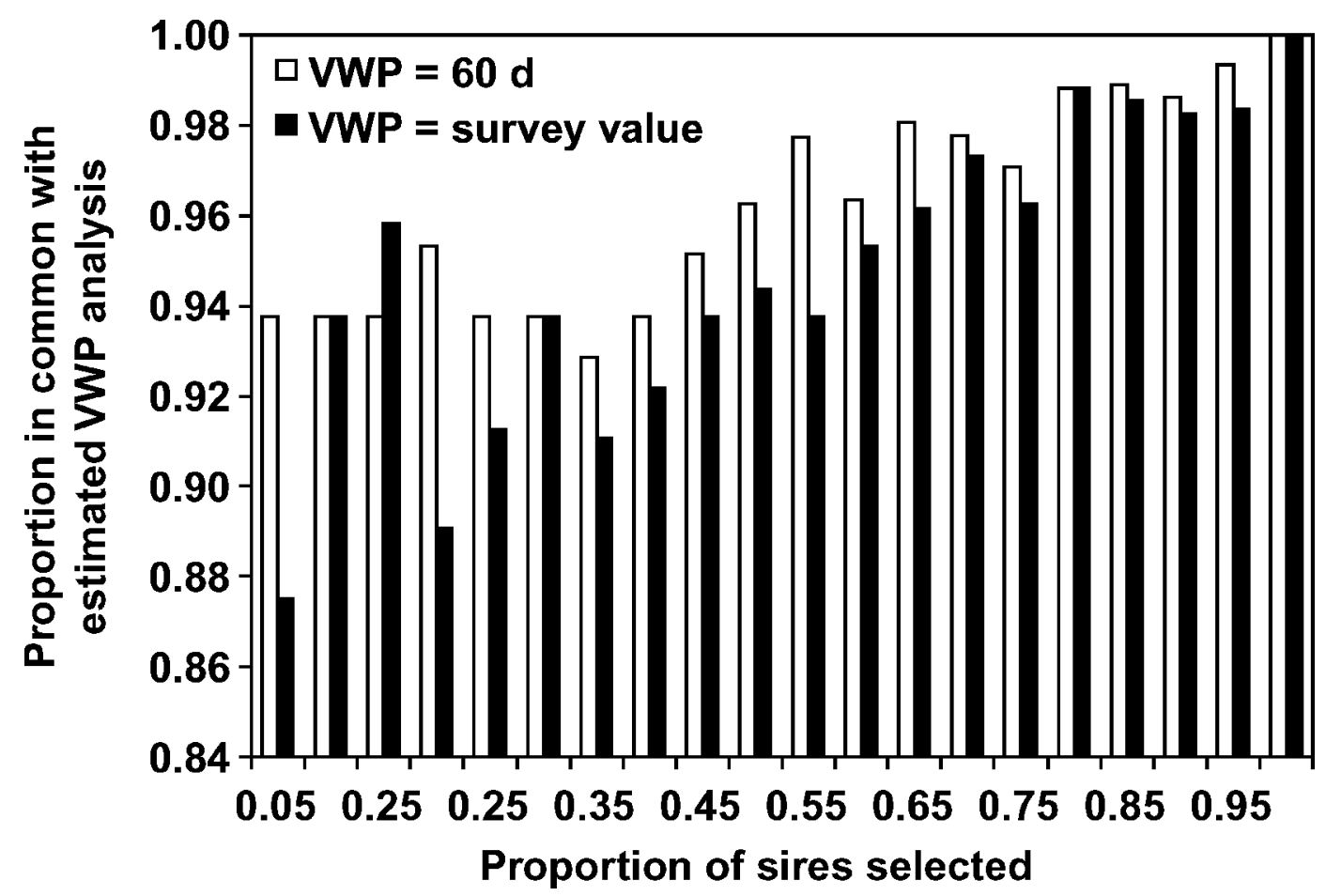

Figure 4. Proportion of sires selected based on sire PTA using estimated posterior means for the voluntary waiting period (VWP) that were also in common with those selected based on sire PTA with the VWP fixed at $60 \mathrm{~d}$ postpartum or the VWP fixed at farmer survey values, from a total population of 325 sires with $\geq 25$ progeny.

ation of dairy sires for the fertility of their lactating daughters. The Gibbs-Metropolis algorithm used in the present study may not be efficient for national genetic evaluations, but a modified Gibbs-Metropolis sampling algorithm could be used to accelerate computations. For example, after the initial burn-in period, both thresholds and VWP parameters could be updated every 100 iterations, and this would significantly reduce computational time. The measure of female fertility used in the present study, namely, the number of 21-d opportunity periods required to achieve pregnancy after the VWP has passed, allows expression of sire PTA in a manner that is consistent with the terminology and software used for reproductive management of modern commercial dairy farms. Differences within farms in duration of the VWP are common, because many farms extend DFS for certain groups of cows based on parity, milk yield, BCS, month of calving, and a variety of other factors. The present study offers a framework for estimating specific VWP parameters for groups of cows within a herd while simultaneously predicting sires' PTA for daughter fertility. Although farmer-reported VWP obtained from a management survey were considered in the present study, it may not be necessary to obtain such information in the future, because VWP estimates obtained from actual data may be more accu- rate than survey values. Future studies should consider alternative strategies for forming "VWP contemporary groups," that is, groups of cows within a herd that are likely to be subject to similar postpartum reproductive management. Such groups could be formed according to parity, milk yield, BCS (or dairy form score), month of calving, or other factors, and optimal grouping strategies may differ according to herd size or geographical location. In this manner, it will be possible to evaluate dairy sires more accurately and fairly, because intentional extension of the VWP for the highest yielding cows within a herd could penalize sires with high-PTA milk and may affect inferences about the genetic correlation between milk yield and female fertility. On the other hand, if a farmer intentionally extends the VWP of a group of cows with high milk yield or low BCS because the perceived likelihood of pregnancy is low, then perhaps some type of penalty is justified. Because estrus events without corresponding inseminations are rarely reported, it is impossible to determine whether extended DFS for an individual cow reflects failure to exhibit estrus or intentional extension of the VWP. Likewise, it is often impossible to determine whether timely DFS for an individual cow reflects naturally occurring estrus or hormonal synchronization. The model proposed herein can account for censoring of records 


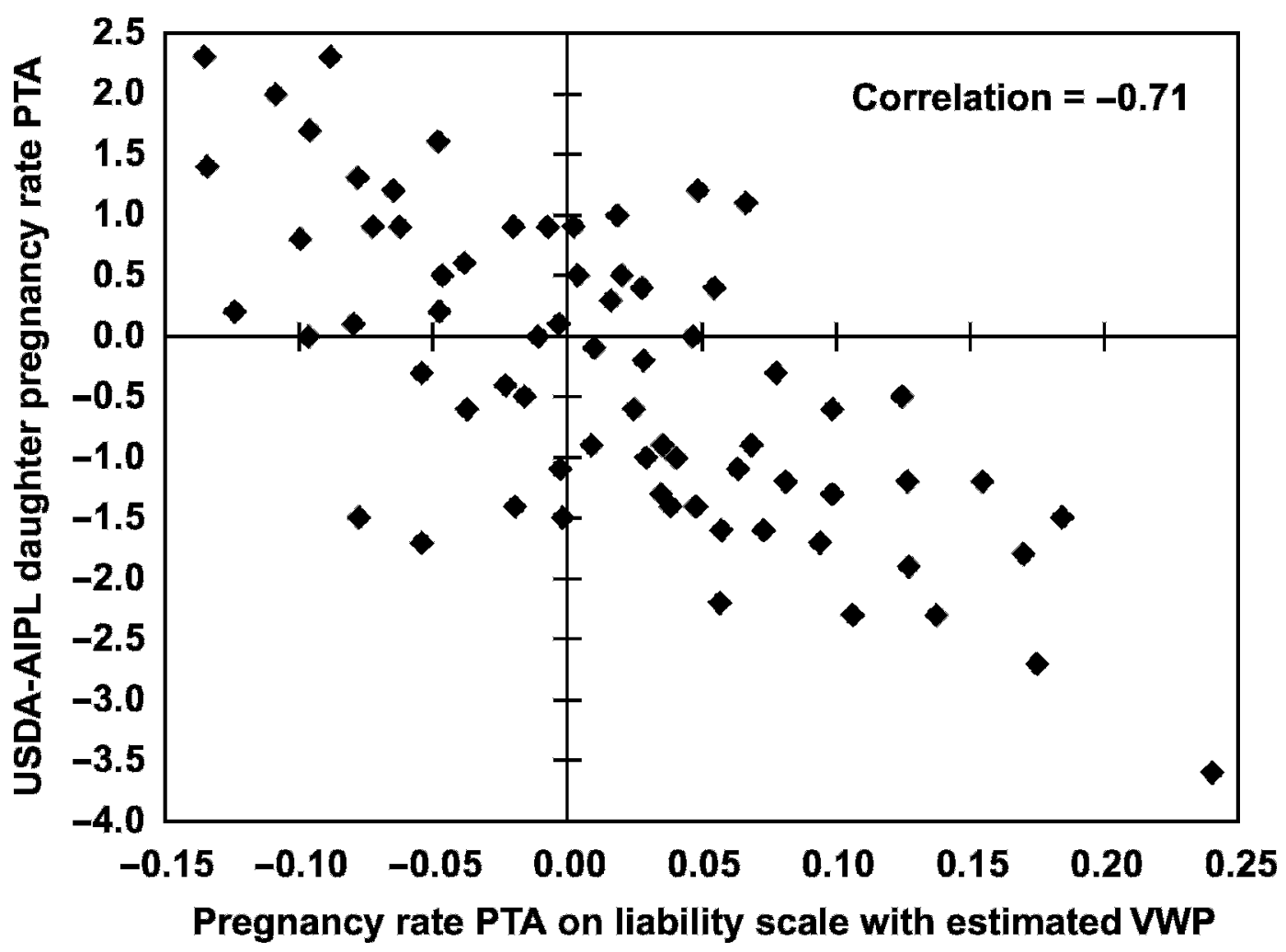

Figure 5. Scatter plot of sire PTA using estimated posterior means for voluntary waiting period (VWP) vs. May 2006 USDA-Animal Improvement Programs Laboratory (AIPL) sire PTA for daughter pregnancy rate, for 71 sires with $\geq 100$ progeny in both data sets.

of cows that fail to conceive prior to completion of 8 opportunity periods, movement to a "clean-up" pen for natural service mating, designation as "do not breed," or culling from the herd, as well as cows that have not yet been diagnosed as pregnant at the time of genetic evaluation. Accommodating censored fertility records is important, whether using an ordinal threshold model or a simple linear model, because ignoring censoring can reduce the precision of sire PTA, and waiting for the subsequent calving can reduce the timeliness of genetic evaluations for this important trait.

\section{ACKNOWLEDGMENTS}

The authors would like to thank the Alta Genetics Advantage Program Consultants for gathering on-farm reproduction data and assisting with completion of the herd management surveys. Cooperation by managers of herds participating at the Alta Genetics Advantage Program is also gratefully acknowledged.

\section{REFERENCES}

Abdallah, J. M., and B. T. McDaniel. 2000. Genetic parameters and trends of milk, fat, days open and body weight after calving in North Carolina experimental herds. J. Dairy Sci. 83:1364-1370.
Albert, J., and S. Chib. 1997. Bayesian Methods for Cumulative, Sequential and Two-Step Ordinal Data Regression Models. Technical Report. Department of Mathematics and Statistics, Bowling Green State University, Bowling Green, $\mathrm{OH}$.

Arbel, R., Y. Bigun, E. Ezra, H. Sturman, and D. Hojman. 2001. The effect of extended calving intervals in high lactating cows on milk production and profitability. J. Dairy Sci. 84:600-608.

Caraviello, D. Z., K. A. Weigel, P. M. Fricke, M. C. Wiltbank, M. J. Florent, N. B. Cook, K. V. Nordlund, N. R. Zwald, and C. L. Rawson. 2006. Survey of management practices on reproductive performance of dairy cattle on large US commercial farms. J. Dairy Sci. 89:4723-4735.

Chebel, R. C., J. E. P. Santos, J. P. Reynolds, R. L. A. Cerri, S. O. Juchem, and M. Overton. 2004. Factors affecting conception rate after artificial insemination and pregnancy loss in lactating dairy cows. Anim. Reprod. Sci. 84:239-255.

Dechow, C. D., G. W. Rogers, L. Klei, T. J. Lawlor, and P. M. VanRaden. 2004. Body condition scores and dairy form evaluations as indicators of days open in US Holsteins. J. Dairy Sci. 87:35343541 .

Dematawewa, C. M. B., and P. J. Berger. 1998. Genetic and phenotypic parameters for 305-day yield, fertility and survival in Holsteins. J. Dairy Sci. 81:2700-2709.

de Vries, A., and C. A. Risco. 2005. Trends and seasonality of reproductive performance in Florida and Georgia dairy herds from 1976 to 2002. J. Dairy Sci. 88:3155-3165.

de Vries, A., C. Steenholdt, and C. A. Risco. 2005. Pregnancy rates and milk production in natural service and artificially inseminated dairy herds in Florida and Georgia. J. Dairy Sci. 88:948956.

Ferguson, J. D., and D. T. Galligan. 1999. Veterinary reproductive programs. Pages 131-137 in Proc. 32nd Annu. Meet. Am. Assoc. Bovine Pract., Nashville, TN. Am. Assoc. Bovine Pract., Auburn, AL. 
González-Recio, O. Y., M. Chang, D. Gianola, and K. A. Weigel. 2006. Comparison of models using different censoring scenarios for days open in Spanish Holstein cows. Anim. Sci. 82:233-239.

Jorjani, H. 2005. Preliminary report of Interbull pilot study for female fertility traits in Holstein populations. Interbull Bull. 33:34-44.

Miller, R. H., H. D. Norman, M. T. Kuhn, and J. S. Clay. 2005. Assessment of voluntary waiting period and frequency of estrus synchronization among herds J. Diary Sci. 88(Suppl. 1):301. (Abstr.)

Oseni, S., I. Misztal, S. Tsuruta, and R. Rekaya. 2003. Seasonality of days open in US Holsteins. J. Dairy Sci. 86:3718-3725.

Oseni, S., S. Tsuruta, I. Misztal, and R. Rekaya. 2004. Genetic parameters for days open and pregnancy rates in US Holsteins using different editing criteria. J. Dairy Sci. 87:4327-4333.

Raftery, A. E., and S. M. Lewis. 1992. How many iterations of the Gibbs sampler? Bayesian Statistics 4: Proc. Fourth Valencia Int. Mtg. J. M. Bernardo, J. O. Berger, A. P. Dawid, and A. F. M. Smith, ed. Oxford University Press, Oxford, UK.

Sorensen, D., and D. Gianola. 2002. Likelihood, Bayesian and MCMC Methods in Quantitative Genetics. Springer-Verlag, New York, NY.
Sterry, R. A., M. L. Welle, and P. M. Fricke. 2006. Effect of interval from timed AI to initiation of resynchronization of ovulation on fertility of lactating dairy cows. J. Dairy Sci. 89:2099-2109.

Stevenson, J. S., J. A. Cartmill, B. A. Hensley, and S. Z. El-Zarkouny. 2003. Conception rates of dairy cows following early not-pregnant diagnosis by ultrasonography and subsequent treatments with shortened Ovsynch protocol. Theriogenology 60:475-483.

Stevenson, J. S., and A. P. Phatak. 2005. Insemination at estrus induced by presynchronization before application of synchronized estrus and ovulation. J. Dairy Sci. 88:399-405.

Tenhagen, B.-A., C. Vogel, M. Drillich, G. Thiele, and W. Heuwieser. 2003. Influence of stage of lactation and milk production on conception rates after timed artificial insemination following $\mathrm{Ov}$ synch. Theriogenology 60:1527-1537.

VanRaden, P. M. 2003. Longevity and fertility trait definitions compared in theory and simulation. Interbull Bull. 30:43-46.

VanRaden, P. M., A. H. Sanders, M. E. Tooker, R. H. Miller, H. D. Norman, M. T. Kuhn, and G. R. Wiggans. 2004. Development of a national genetic evaluation for cow fertility. J. Dairy Sci. $87: 2285-2292$. 